\title{
DiFERENCIAS DE CONOCIMIENTOS, VALORACIÓN Y USO DE Cactáceas entre pobladores de Salinas Grandes Y Sistema Serrano (Córdoba, Argentina)
}

\author{
DIFFERENCES IN KNOWLEDGE, VALUATION AND USE OF CACTI AMONG \\ Residents of Salinas Grandes and Serrano System (Córdoba, \\ Argentina)
}

\author{
Julieta K. Torrico Chalabe ${ }^{1,2}$ y Cecilia Trillo2
}

\begin{abstract}
1. Becaria Doctoral Consejo Nacional de Investigaciones Científicas y Técnicas (CONICET) 2. Departamento de Diversidad Biológica y Ecología, FCEFyN, UNC e IMBIV (Instituto Multidisciplinario de Biología Vegetal)

*jullieta_111@hotmail.com

Citar este artículo TORRICO CHALABE, J. K. \& C. TRILLO. 2019. Diferencias de conocimientos, valoración y uso de Cactáceas entre pobladores de Salinas Grandes y Sistema Serrano (Córdoba, Argentina). Bol. Soc. Argent. Bot. 54: 125-136.
\end{abstract}

DOI: http://dx.doi. org/10.31055/1851.2372.v54. $\mathrm{n} 1.23590$

Recibido: 17 Agosto 2018 Aceptado: 18 Diciembre 2018 Publicado: 28 Marzo 2019 Editora: Norma Hilgert (iD

ISSN versión impresa 0373-580X ISSN versión on-line 1851-2372

\section{SUMMARY}

Background and aims: The valuation, use and management practices of natural resources of small producers, in general rely on local ecological knowledge (LEK). In order to compare the communities of two physiographic units: Salinas Grandes and Serrano System, the study of LEK on cacti was discussed.

M\&M: We performed 21 interviews with key informants about diversity, uses and valuation.

Results: On the one hand, in Salinas Grandes one use and management practice associated with traditions for each taxon were mentioned at least; on the other hand, less types of uses were mentioned by Serrano System's informants.

Conclusions: In this place, uses related to tourism and commercialization highlight. Residents of Salinas Grandes, who live in extreme aridity conditions and have scarce herbaceous resources available, conserve knowledge linked to traditions which is necessary to ensure their social reproduction, and the Cactaceae appear as key resources for their subsistence. Those of the Serrano System, which have a greater diversity of forage resources, can offer other services and reorient their knowledge towards new activities. This suggest the flexibility of this knowledge (LEK), which allows adjustments to deal with contextual change situations.

\section{KEY WORDS}

Cactaceae, changes, ethnobotany, knowledge, small producers, uses.

\section{RESUMEN}

Introducción y objetivos: La valoración, uso y manejo de los recursos naturales por parte de pequeños productores se sustenta en el conocimiento ecológico local (CEL). Con el objeto de comparar entre sí las comunidades de dos unidades fisiográficas: Salinas Grandes y Sistema Serrano, abordamos el estudio del CEL sobre las cactáceas.

M\&M: Realizamos 21 entrevistas a informantes claves sobre diversidad, usos y valoración.

Resultados: En Salinas Grandes nos mencionaron por lo menos un uso para cada taxón y formas de manejo asociadas a tradiciones; en cambio, los informantes del Sistema Serrano mencionaron menos tipos de usos y cobraron relevancia otros tipos relacionados al turismo y la comercialización.

Conclusiones: Por un lado, los pobladores de Salinas Grandes, que viven en condiciones de aridez extrema y con escasos recursos herbáceos, conservan conocimientos ligados a tradiciones que son necesarios para asegurar su reproducción social, donde las Cactáceas aparecen como recursos claves para su subsistencia. Por otra parte, los del Sistema Serrano, que cuentan con mayor diversidad de recursos forrajeros, pueden ofrecer otros servicios y reorientan sus conocimientos hacia nuevas actividades, donde se evidencia la flexibilidad de estos saberes (dentro del CEL) que permite ajustes a las situaciones de cambio contextual.

Palabras clave

Cactáceas, cambios, conocimientos, etnobotánica, pequeños productores, usos. 


\section{INTRODUCCIÓN}

Los sistemas de conocimiento locales, según Vandebroek et al. (2011), consisten en conjuntos de saberes y creencias, en definitiva, visiones del mundo desarrolladas y sostenidas por las comunidades humanas. En términos de Nazarea (1999), es el sistema de conocimiento situado de las personas que participan de una trama de relaciones con su entorno, localizada en el tiempo y el espacio. En este marco se inscribe el denominado conocimiento ecológico local (CEL) (Berkes, 1999; Correa et al., 2012), referido a las relaciones entre las personas y su entorno biológico y cultural, que orienta, por ejemplo, la valoración, selección y manejo de los recursos naturales locales: lo que es un recurso para un determinado grupo humano puede no serlo para otro (Hurrell et al., 2018).

El CEL no es estático sino dinámico: cambia de acuerdo a los ajustes del grupo humano, a las circunstancias cambiantes de su entorno biocultural (Hurrell, 2014). El término biocultural, en este contexto, se desprende del concepto de diversidad biocultural, es decir, la diversidad de la vida tanto en sus aspectos biológicos como culturales, aspectos que no se asumen como instancias separadas, o que transcurren por vías paralelas; por el contrario, interactúan de forma compleja y co-evolucionan (Maffi, 2001, 2005).

En diversos estudios etnobotánicos sobre Cactáceas (Caballero et al., 1998; Caballero \& Cortés, 2001; Casas et al., 1987; Casas, 2001), se destaca su valoración positiva por parte de los pobladores locales debido a su capacidad de persistir en condiciones de extrema aridez, así como su importancia cultural, expresada en una gran diversidad de usos y un mejor aprovechamiento como recurso.

En la Argentina, existen múltiples referencias etnobotánicas sobre Cactáceas en el marco de estudios más amplios (Martínez-Crovetto, 1964; Roig \& Bárcena, 1983; Arenas \& Scarpa, 1998; Biurrun et al., 2007; Scarpa, 2007, Arias Toledo \& Trillo, 2014), en los que se relevaron sus usos tradicionales como alimento, forraje, remedios caseros y colorantes. En la provincia de Córdoba, se destacan los trabajos de Herrera (2003) y Zapata et al. (2005). El uso de estas plantas y su valoración histórica por parte de los pobladores del norte de Córdoba queda evidenciado por el hallazgo de restos arqueológicos en el noroeste de esta provincia, que muestran la utilización del "cardón" (Stetsonia coryne (Salm-Dyck) Britton \&
Rose) como cercos vivos (Berberián, 1987; Pastor et al., 2012; Recalde, 2015). Además, en trabajos etnobotánicos en esta zona (Torrico Chalabe \& Trillo, 2015; Ahumada \& Trillo, 2017), se han indicado numerosos usos actuales nuevos (como el ornamental, recurso estético y para la comercialización) y valoraciones positivas asociadas a estas plantas, donde se destacan "tuna", Opuntia ficus-indica (L.) Mill., con muchas variedades locales; "cardón", "quimilo", Opuntia quimilo K. Schum., y "tunilla", Opuntia sulphurea Gillies ex Salm-Dyck.

En la provincia de Córdoba, una de las principales problemáticas bioculturales se encuentra íntimamente relacionada a la producción agropecuaria, la que presenta diferentes niveles de intensidad y que, a lo largo de las últimas décadas, ha sufrido importantes procesos de deforestación, desertización y “descampesinización” (Cáceres et al., 2010; Cáceres, 2014), en relación al avance de la frontera agraria (Kimbrell, 2002). El cambio en el uso de la tierra y de las tecnologías de producción se destacan como mayores modificaciones en el Sistema de Bosque Serrano en comparación con Salinas Grandes, que no presenta cambios considerables (Cabido et al., 2005, Hoyos et al., 2012).

En el norte provincial, y en mayor intensidad en las zonas de Sistema Serrano y de planicies que presentan una mayor aptitud productiva, la pérdida de biodiversidad y la desaparición de la mayoría de los agricultores tradicionales se vinculan al avance de la agricultura industrial, entre otros factores, que conlleva una pérdida en el sistema de conocimientos locales tradicionales, producto de años de interacción entre las personas y su entorno vegetal (Trillo et al., 2007; Arias Toledo et al., 2010; Arias Toledo \& Trillo, 2014).

A partir de estudios previos sobre la importancia de las Cactáceas en la vida de las poblaciones de las zonas áridas de Córdoba (Torrico Chalabe \& Trillo, 2015; Ahumada \& Trillo, 2017), surgió el interés de comparar y analizar el conocimiento ecológico local (CEL) sobre las Cactáceas. El trabajo se enfocó en las narrativas de los informantes claves de diferentes comunidades criollas de las unidades fisiográficas de Salinas Grandes (localidades alejadas de los principales centros urbanos) y Sistema Serrano (contextos pluriculturales cercanos a los centros urbanos y con desarrollo turístico).

Las principales preguntas que orientaron el trabajo fueron: ¿existen diferencias en cuanto a 
los saberes sobre los cactus entre los informantes de Salinas Grandes y del Sistema Serrano? ¿Qué relevancia tienen los conocimientos ligados a tradiciones en ambas unidades fisiográficas para estos informantes de comunidades rurales?

\section{MATERIALEs Y MÉTOdos}

Área de estudio

El trabajo se realizó en 12 localidades del noroeste de la provincia de Córdoba, que corresponden a la Provincia Fitogeográfica Chaqueña, en las subregiones (Fig. 1A-B) de Chaco Árido y Chaco Serrano (Cabrera, 1976; Atlas de Bosques Nativos de Argentina, 2003), y que también se enmarcan en las zonas fisiográficas de Salinas Grandes y Sistema Serrano (Fig. 1C) (Cabido \& Zak, 1999).

Las Salinas Grandes presentan precipitaciones entre 300 y $500 \mathrm{~mm}$ anuales mientras que el Sistema Serrano entre 400 y $800 \mathrm{~mm}$ por año, ambas zonas con un máximo de lluvias en verano, que concentra el $70 \%$ de la precipitación total (Cabrera, 1976); mientras que la temperatura

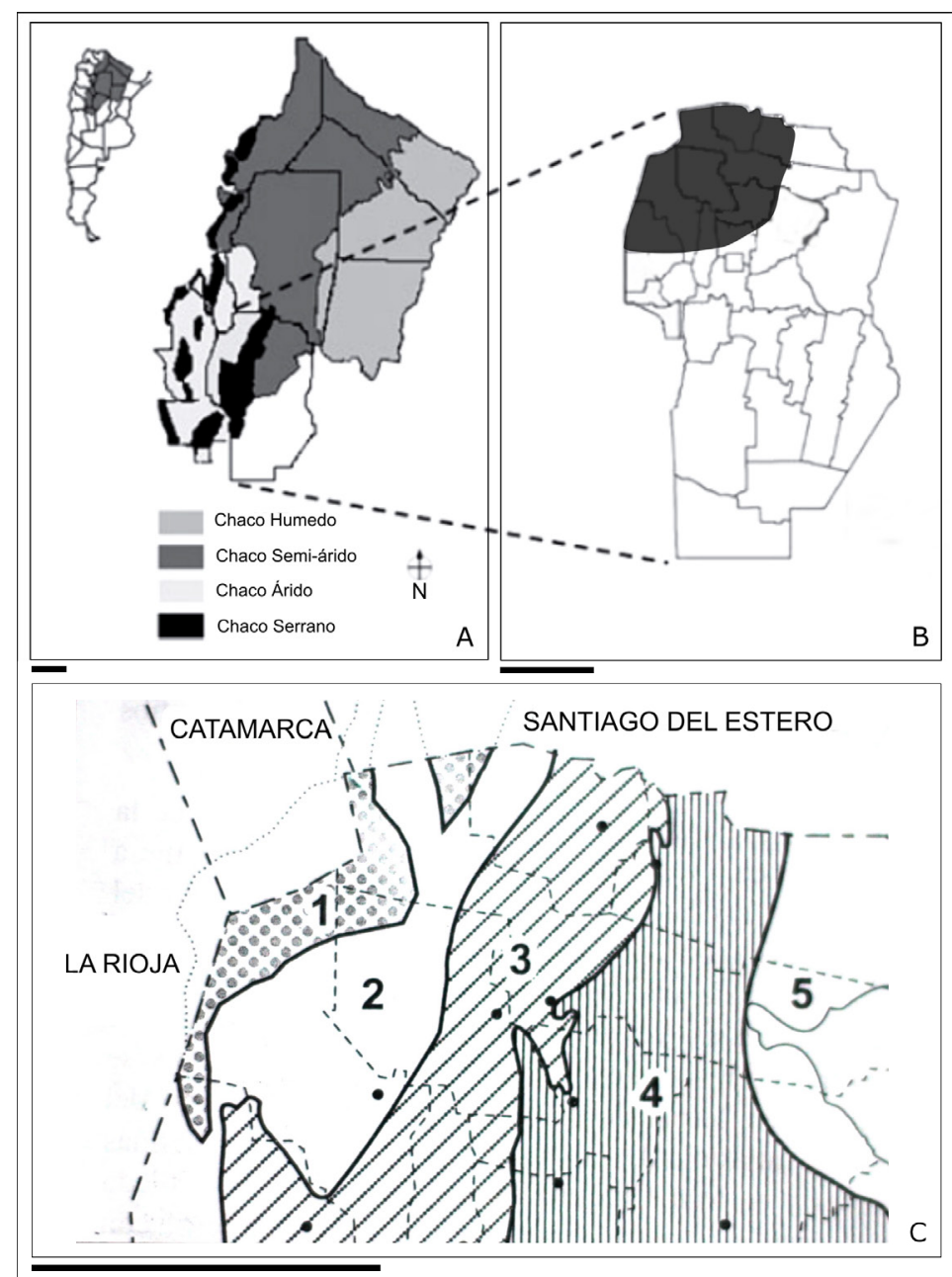

Fig. 1. Área de estudio. A: Localización del Gran Chaco y de la provincia de Córdoba, Argentina, y las subregiones del Gran Chaco (Atlas de Bosques Nativos de Argentina, 2003). B: Zona de estudio en el norte de Córdoba. C: Zonas fisiográficas del norte de Córdoba 1-Salinas Grandes, 2-Planicie Occidental, 3-Sistema Serrano, 4-Planicie Oriental y 5-Depresión de Mar Chiquita (Cabido \& Zak, 1999). Escalas= $140 \mathrm{~km}$. 
media anual aumenta en sentido contrario $\left(18,6^{\circ} \mathrm{C}\right.$ de temperatura media anual en Sistema Serrano y $19,9^{\circ} \mathrm{C}$ en Salinas Grandes) (Cabido \& Zak, 1999). Ambas zonas presentan un balance negativo entre la precipitación y la evapotranspiración, y la mayor parte del año hay déficit de agua (FAO, 2007). Como consecuencia, el invierno es particularmente duro para la vida de las personas y de los animales, y es casi imposible establecer cultivos como por ejemplo de secano.

Las localidades del Sistema Serrano en las que se trabajó son: San Marcos Sierra (2199 habitantes), Cerro Colorado (285), Guasapampa (241), La Playa (220), Chañar Viejo (186), Eufrasio Loza (166) y Rayo Cortado (691) (INDEC, 2010) (Fig. 2). Esta zona presenta una cubierta vegetal que se distribuye en pisos o cinturones altitudinales de vegetación: bosques entre los 700 y $1200 \mathrm{msnm}$; matorrales entre los 900 y $1500 \mathrm{msnm}$; pastizales por encima de los $1500 \mathrm{msnm}$. Comprende 3 unidades vegetación: 1) bosque serrano, con una fisonomía de bosque abierto a semi-cerrado, 2) matorrales serranos o "romerrillares", dominados por el "romerillo", Baccharis aliena (Spreng.) Joch. Müll., 3) un "espinillar", donde domina el "espinillo", Vachellia caven (Molina) Seigler \& Ebinger. La mayor parte del área ha sido alterada de forma directa o indirecta por las actividades humanas, considerándose zonas de "vegetación cultural", es decir, una zona de flora natural que ha sufrido cambios significativos en su composición y estructura (Cabido \& Zak, 1999; Cabido \& Pacha, 2002). Las principales actividades económicas de estas ciudades son el turismo y la producción ganadera para autosustento y ventas menores (Cáceres et al., 2006). Estas poblaciones se constituyen en contextos pluriculturales, dado que las personas provienen de otras áreas urbanas del país y de la provincia, y también de áreas rurales cercanas; estos pobladores son descendientes de inmigrantes $y / o$ de poblaciones originarias de la zona. Se considera que, desde el punto de vista de los saberes locales, en el contexto pluricultural coexisten conocimientos ligados a las tradiciones de origen y los no tradicionales, novedosos, difundidos en la urbanización creciente y en los medios de comunicación (Hurrell, 2014).

Las localidades de las Salinas Grandes en las que se trabajó son: Serrezuela y Piedrita Blanca (2678 habitantes), La Batea (180), San José de las Salinas (777) y Lucio Victorio Mansilla (881) (INDEC,

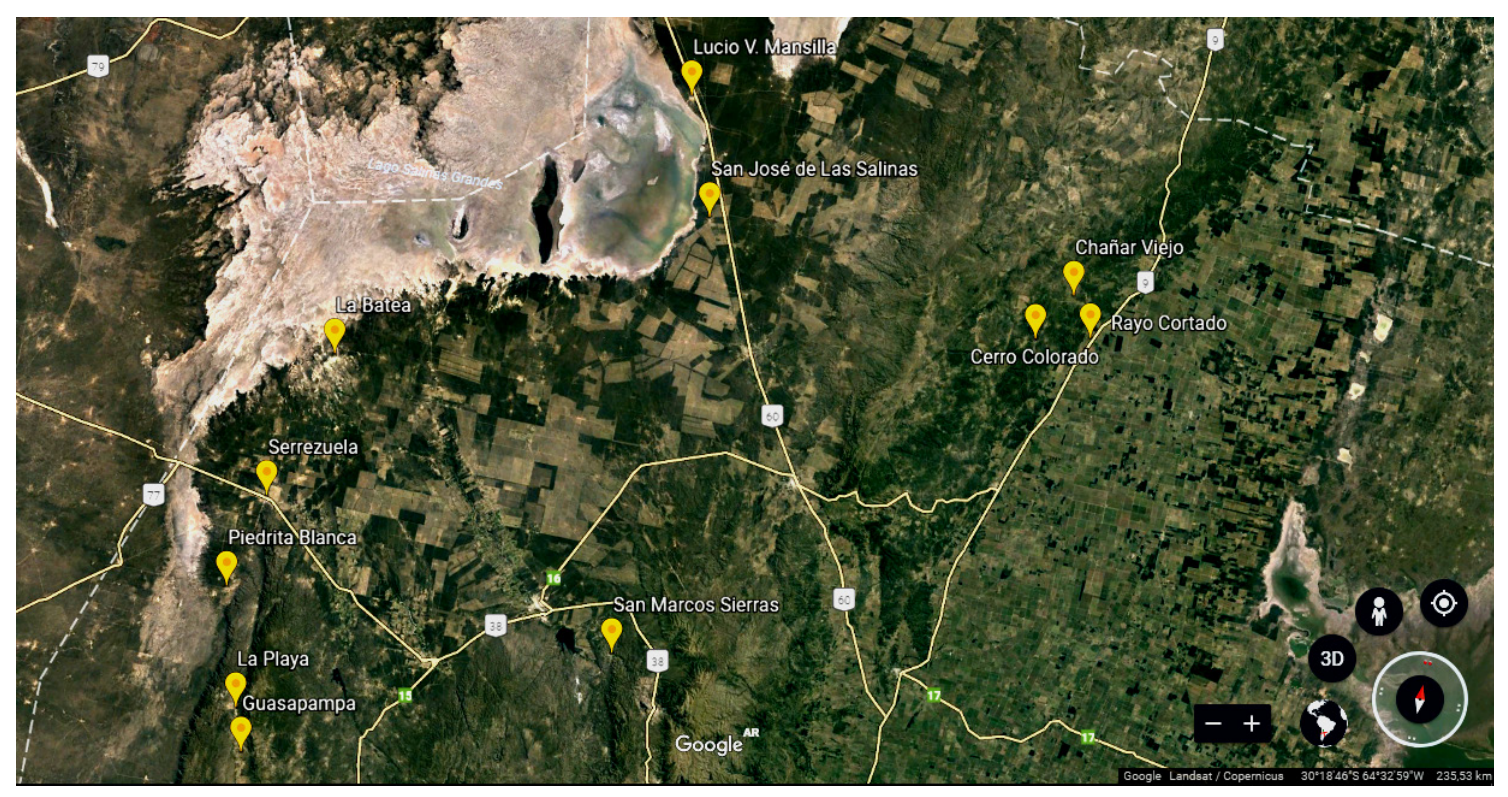

Fig. 2. Ubicación del área de estudio, con identificación de las localidades visitadas: San Marcos Sierra, La Playa, Cerro Colorado, Chañar Viejo y Rayo Cortado (Sistema Serrano) y Serrezuela, La Batea, Piedrita Blanca, San José de las Salinas y Lucio V. Mansilla (Salinas Grandes) (Imagen Satelital de Google Earth, 2017). 


\section{J. K. Torrico Chalabe y C. Trillo - Conocimientos, valoración y usos de Cactáceas}

2010) (Fig. 2). La depresión de las Salinas Grandes se encuentra entre 150 y $300 \mathrm{msnm}$, el suelo de este "bolsón" o "playa" es relativamente plano y salino, conformando característicos desiertos de sal con manchones de vegetación halófita; los márgenes, más elevados, presentan una vegetación transicional entre los matorrales de plantas halófitas y el bosque chaqueño xerófito (Cabido \& Zak, 1999). La vegetación halófita incluye dos unidades: 1) el matorral halófito bajo, o "jumeal" de Allenrolfea patagónica (Moq.) Kuntze y Heterostachys ritteriana (Moq.) Ung.-Sternb., y 2) el matorral sub-halófito bajo, con "cardón" (Stetsonia coryne). La principal actividad económica es la cría de ganado ovino caprino el área es de difícil acceso, con condiciones climáticas muy radicales y está alejada de las principales urbes de la zona (Cáceres et al., 2006).

\section{Metodologías etnobotánicas y análisis de datos}

Para la realización de este trabajo se siguieron metodologías habituales en etnobotánica: entrevistas, recorridas etnobotánicas y observación participante (Albuquerque et al., 2014, 2018). En todos los casos se obtuvo el consentimiento oral informado, de acuerdo a lo establecido en el Código de Ética de la SOLAE (2016) y se establecieron mecanismos claros, precisos y previamente acordados de reciprocidad con los pobladores con quienes se trabajó. Se hicieron relevamientos preliminares sobre la familia Cactaceae en el norte de córdoba (Torrico Chalabe \& Trillo, 2015; Ahumada \& Trillo, 2017) y posteriormente se desarrolló un proyecto de extensión en conjunto con la Agencia de Extensión Rural Villa de María del Río Seco (INTA): "Puesta en valor del conocimiento y germoplasma de los cactus del Norte de Córdoba. Intercambio recíproco y acciones de conservación con pobladores y gestores" (Resolución UNC: 1428/2014-HCS).

Los actores sociales del área de estudio incluyen técnicos, pequeños, medianos y grandes productores. Este estudio se enfocó en los pequeños productores y campesinos, autoconsiderados "criollos" (Trillo, 2010). El pequeño productor campesino posee una unidad agropecuaria de producción y consumo, bajo distintas formas de tenencia, que por la escasez de recursos naturales o de capital se basa principalmente en el grupo familiar (Bergamín, 1992; Trillo, 2010). Cáceres et al. (2006) sostienen que las "unidades campesinas" del noroeste de la provincia de Córdoba pueden entenderse como ámbitos de actividad múltiple, no restringidas exclusivamente al campo productivo.

La aproximación realizada fue cuali-cuantitativa, e incluyó el análisis de las narrativas obtenidas en entrevistas libres y semi-estructuradas (Fernandes Saraiva, 2012; Parlee et al., 2012; Pochettino \& Hurrell, 2013; Albuquerque et al., 2014). En las recorridas con informantes y observación participante se recurrió a la utilización de un álbum de fotografías de las especies de la zona (Torrico Chalabe \& Trillo, 2015). Se realizó una muestra no aleatoria y sólo se incluyeron informantes clave, es decir, pobladores criollos, pequeños productores y campesinos con un profundo conocimiento de la familia Cactaceae (Costa-Neto, 2002). El método de muestreo fue el de "bola de nieve", en el cual el primer sujeto contactado da al investigador el nombre de otro sujeto, que a su vez proporciona el de un tercero, y así sucesivamente (Aguilera et al., 2003).

Se realizaron entrevistas a 8 y 13 informantes claves pertenecientes a Salinas Grandes y al Sistema Serrano, respectivamente (Padua, 1994; Aldrige \& Lavine, 2003; Bernard, 1995). En todas se realizaron preguntas generales (Cotton, 1996) sobre los usos de las Cactáceas (actuales o pasados, si eran anecdóticos o novedosos), los taxones reconocidos (cómo se identifican y cuál es su manejo en sus campos) y sobre la valoración de la familia Cactaceae en general (positiva o negativa para su vida diaria, importancia de algunos taxones, y relatos sobre su rol actual).

Las especies, subespecies y formas de Cactaceae relevadas (12 taxones en total) fueron registradas mediante fotografías. Su identificación botánica fue realizada a partir de bibliografía específica (Kiesling \& Ferrari, 2005; Demaio \& Trevisson, 2006; Torrico Chalabe \& Trillo, 2015) y la nomenclatura científica fue actualizada consultando la base de datos del Catálogo de Plantas Vasculares del Conosur.

Se realizaron histogramas de frecuencia relativa para mostrar tanto la cantidad de informantes que reconocieron cada taxón, como también la cantidad de informantes que mencionaron los diferentes tipos de uso; comparando ambas zonas fisiográficas.

A partir de las narrativas, se realizó un cuadro comparativo basado en 2 criterios de comparación: a) temporalidad de usos, b) valoración de las Cactáceas. La temporalidad de los usos, permitió analizar si eran actuales o pasados (anecdóticos) y evaluar cómo se genera el proceso de resignificación. Se buscó relevar, en particular, los relatos sobre los usos asociadas a 
conocimientos tradicionales, cuestionando si existían en la actualidad, cómo se hubieran resignificado o si se los nombraba como un recuerdo. Para la segunda categoría, se tomó como criterio cuáles eran los saberes más importantes para cada unidad, a que motivación refería esa relevancia y por la valoración general de las Cactáceas.

\section{Resultados}

Análisis cuantitativo

En las gráficas (Figs. 3, 4) se observan las frecuencias relativas sobre el reconocimiento de los 12 taxones trabajados y sobre el conocimiento de usos que poseen los informantes claves.

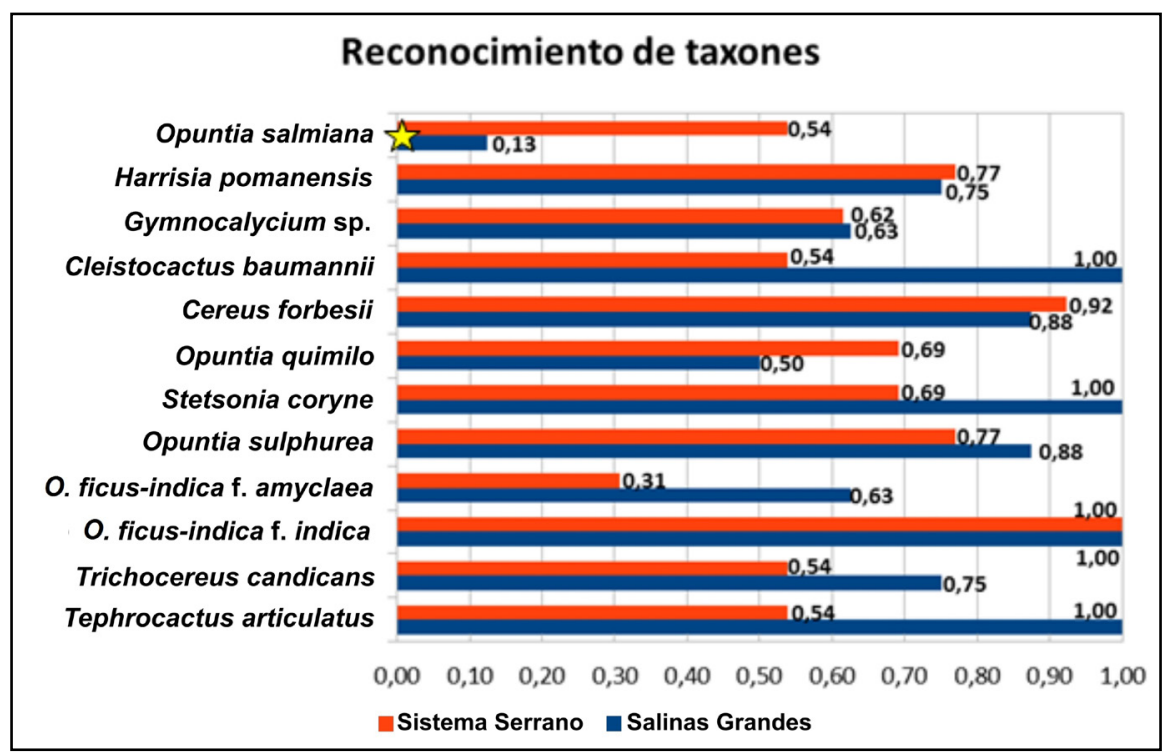

Fig. 3. Histograma de frecuencias relativas de reconocimiento sobre la totalidad de entrevistas en cada zona (eje X) de cada taxón de Cactáceas (eje Y). *Se destaca Opuntia salmiana que sigue un patrón inverso.

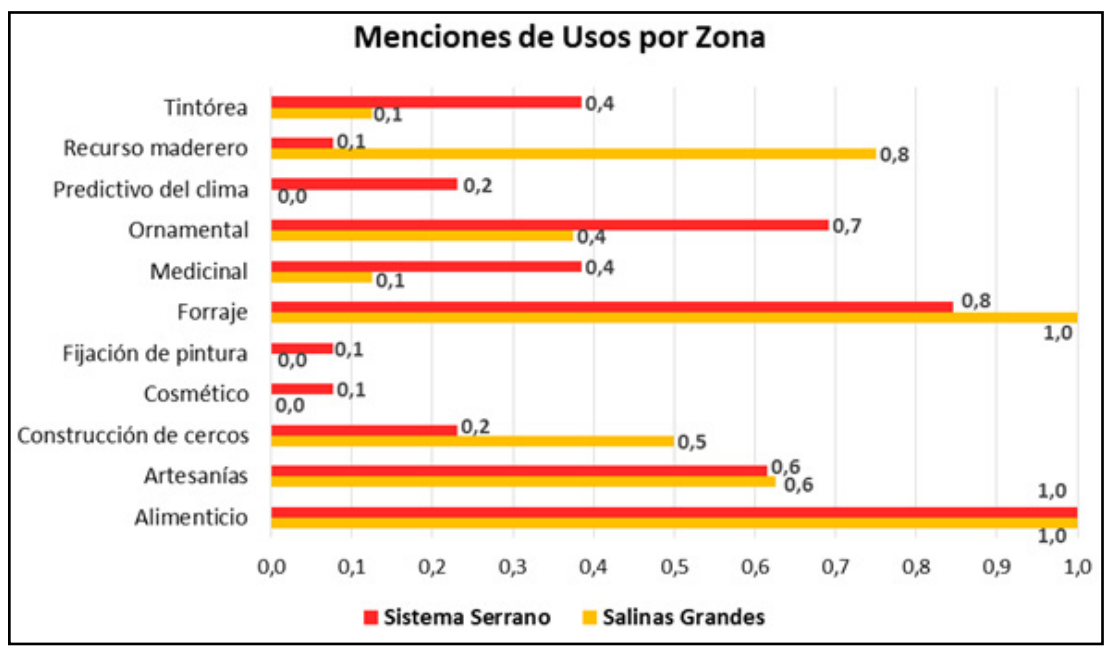

Fig. 4. Histograma de frecuencias relativas de informantes que mencionaron tipos de usos para algún taxón de Cactáceas sobre la totalidad de entrevistas en cada zona (eje $\mathrm{X}$ ) de cada tipo de uso (eje Y). 


\section{J. K. Torrico Chalabe y C. Trillo - Conocimientos, valoración y usos de Cactáceas}

De manera general, se puede observar que en Salinas Grandes (SG) es mayor la cantidad de personas que poseen conocimientos de los taxones que en el Sistema Serrano (SS) (Fig. 3) y que todos los informantes mencionaron al menos un tipo de uso alimentario para cada taxón reconocido (Fig. 4). Se destaca como excepción a Opuntia ficusindica, ya que todos los entrevistados la reconocen y mencionan sus usos. También es importante resaltar que, a pesar de las diferencias registradas en la cantidad de informantes que las reconocen, todas las especies fueron nombradas por lo menos una vez en ambas zonas fisiográficas y que los entrevistados las mencionan como parte de la vegetación nativa de la zona.

En cuanto al reconocimiento de los taxones (Fig. 3), todos fueron identificados en ambas zonas, y se presenta como caso particular la "bola de gato" (Opuntia salmiana Parm.) que es más reconocida por los pobladores del Sistema Serrano (54\% SS y $13 \%$ SG). Este taxón, que además es reconocido como el más abundante de la zona, también es nombrado "huevo de gato" y como "la que hace llorar al tigre" ("uturungo huakachinan" en quichua) (Demaio \& Trevisson, 2006; Di Lullo, 1946). Esta última forma de identificación tiene un alto peso cultural por su historia relacionada al "tigre" Panthera onca L., un relato oral que se trasmitió generación a generación, dado que el último ejemplar en la provincia fue cazado en 1958 en la zona de Bañados del río Dulce (Haro et al., 2006), pero continúa presente en las narrativas actuales, como sucede con los animales domésticos y de cría en su vida diaria. "La planta que hace llorar al tigre, se le dice así porque estando en el monte, al tigre se le pego en la mano el frutito, se lo quiso sacar con la boca, y luego en la mano, y eso es lo que lo hace llorar, cuando se le pega a los perros también lloran" (Mistol F., 66 años, Varón). En cuanto a Salinas Grandes, hay 4 taxones que fueron reconocidos por todos los informantes, la "colita de zorro" (Cleistocactus baumannii), el “cardón" (Stetsonia coryne), la "tuna" (Opuntia ficus-indica f. ficus-indica) y el "huevo de indio" (Tephrocactus articulatus). De estas últimas, se mencionan usos forrajeros, alimentarios y para construcción de cercos, mientras que al "huevo de indio" es reconocida por ser una especie negativa para el ganado.
En cuanto a los tipos de usos (Fig. 4) en ambas zonas los más mencionados son el uso alimentario y para forraje, y también se pueden observar algunas diferencias, por ejemplo: en Sistema Serrano el tercer uso más mencionado es el ornamental, mientras que en Salinas Grandes se indica como recurso maderero.

\section{Análisis comparativo de las narrativas}

Se realizó un cuadro comparativo a partir de las narrativas de los informantes clave y se añadieron algunas expresiones de los entrevistados como ejemplos (Tabla 1).

\section{Discusión y Conclusiones}

Este trabajo permitió comparar las unidades fisiográficas chaqueñas de Salinas Grandes y Sistema Serrano del Noroeste de Córdoba, en relación con la valoración y los usos de las "pencas" (plantas del género Opuntia), "tunas" (variedades o frutos de Opuntia ficus-indica) y "cactus" (nombre común general para las Cactaceae), correspondientes a 10 especies nativas y 2 formas de Opuntia ficus-indica naturalizada, que forman parte de la vida local de los pobladores entrevistados.

La unidad fisiográfica Salinas Grandes se destacó por el alto reconocimiento de las especies nativas de Cactáceas y por el mayor conocimiento de usos asociados a la subsistencia. Al igual que Ruiz Posse et al. (2007), se observó en particular que las Cactáceas en esta zona constituyen un recurso de gran importancia para el desarrollo y reproducción social de las comunidades locales. Esto también explica cómo tanto las prácticas de manejo (Torrico Chalabe \& Trillo, 2015) como los usos ligados a tradiciones se preservan en la actualidad (Cavanna et al., 2010). Al igual que en otros trabajos (Cavanna et al., 2010; Pastor et al., 2012; Recalde, 2015), Stetsonia coryne resulta una especie característica en las zonas de Salinas Grandes, principalmente por su consideración como recurso forrajero clave (Cavanna et al., 2009; Coirini et al., 2010; Karlin et al., 2013).

En la unidad fisiográfica Sistema Serrano, los pobladores entrevistados reconocen la mayoría de las especies y conocen sus nombres vernáculos, como se indicó en trabajos previos (Torrico Chalabe \& Trillo, 2015), pero los conocimientos sobre los 


\section{Bol. Soc. Argent. Bot. 54 (1) 2019}

Tabla 1. Diferencias entre las narrativas de informantes claves de Salinas Grandes y Sistema Serrano.

\begin{tabular}{|c|c|c|}
\hline Criterios & Salinas Grandes & Sistema Serrano \\
\hline Temporalidad de los de usos & $\begin{array}{l}\text { En las narrativas de los informantes se destaca que todos } \\
\text { los usos de los diferentes taxones se mencionan como usos } \\
\text { actuales, que forman parte de su vida cotidiana. } \\
\text { El quishcaloro es el que más se usa después del cardón, el } \\
\text { fruto se come y hacemos arrope, y se le da a la vaca toda la } \\
\text { planta, en tiempos de seca tiene valor. (Omar S. } 64 \text { años, } \\
\text { Varón). } \\
\text { Con el ucle [Cereus forbesii Otto ex C.F. Först.], hacemos } \\
\text { arrope, dulce es, y se usa de forraje y con el ucle rojo [Cereus } \\
\text { aethiops Haw.] también.(Isabel O., } 62 \text { años, Mujer) } \\
\text { La úluva [Cleistocactus baumannii (Lem.) Lem.] se come el } \\
\text { fruto y se usa para forraje.(Isabel O., } 62 \text { años, Mujer). } \\
\text { Además se mencionan como actuales y relevantes, usos } \\
\text { asociados a tradiciones. } \\
\text { Las pencas como siempre se usan como forraje, quemamos } \\
\text { las espinas y se las damos a los animales. (Norberto L. } 63 \\
\text { años, Varón). } \\
\text { El cardón la flor y la fruta la usamos de forraje, así lo hacía } \\
\text { desde mi abuela. La madera del cardón para el horno y para } \\
\text { hacer artesanías. Se construye el cerco de cardón. (Perla L. } \\
61 \text { años, Mujer). }\end{array}$ & $\begin{array}{l}\text { Se indican diversas alusiones a usos ligados a tradiciones, } \\
\text { como parte del pasado que, en este momento, perdieron } \\
\text { actualidad. } \\
\text { La tuna, es la que más se utiliza, para el arrope, y antes se } \\
\text { la partía al medio para usar la babasa para sacar el calor, es } \\
\text { una planta fría, que se usaba para la indigestión. (Marta B., } \\
69 \text { años, Mujer). } \\
\text { La surpiña o surpiyar [Cleistocactus baumannil], a la fruta la } \\
\text { comíamos de chicos, así nos criaron, es lindo recordar eso, } \\
\text { que cuando voy a ver a mis amigos a Córdoba, les llevo. } \\
\text { (Mistol F., } 66 \text { años, Varón). } \\
\text { A las vacas en el invierno antes les cortábamos la penca para } \\
\text { alimento, se usaba mucho. (Griselda l., } 43 \text { años, Mujer). }\end{array}$ \\
\hline Valoración de las Cactáceas & $\begin{array}{l}\text { Son los relacionados con la subsistencia de las personas y } \\
\text { de la ganadería; por esto se destacan los usos para forraje, } \\
\text { alimentación y construcción de cercos. } \\
\text { El cardón es el más valioso, para el que es ganadero más que } \\
\text { nada, porque se le da todo, la penca, la flor se las bajamos, la } \\
\text { usamos para armar los cercos y también para leña. Además, } \\
\text { para la sed del que trabaja. (Omar S. } 64 \text { años, Varón } \\
\text { Los cactus son valiosos para los animales, son muy útiles y } \\
\text { más para los tiempos de seca. (Domingo R., } 55 \text { años, Varón) } \\
\text { La valoración generalmente es positiva centrada en su } \\
\text { importancia como recurso de subsistencia. } \\
\text { Los cactus tienen mucho valor por los animales, porque es el } \\
\text { alimento. (Esteban B., } 66 \text { años, Varón). } \\
\text { Son útiles porque cuando hay sequías es lo que te ayuda a } \\
\text { salir del invierno. (Noberto L., } 63 \text { años, Varón) } \\
\text { Es bueno que haya por los animales, las pencas, son muy } \\
\text { importantes, más en agosto y septiembre, que escasea. (Irma } \\
\text { V. } 60 \text { años, Mujer). } \\
\text { Se destaca una especie entre las demás, el "cardón" } \\
\text { (Stetsonia coryne), por su multiplicidad de usos y de dar } \\
\text { respuesta a muchas de sus necesidades. } \\
\text { El cardón es la penca de las sequías, te da fruto y flor para } \\
\text { el animal y la pala para calmarle la sed, se usa también para } \\
\text { el fuego. Y los frutos se comen. (Noberto L., } 63 \text { años, Varón) } \\
\text { El cardón se usa para varias cosas, el fruto y la flor se la baja } \\
\text { para los animales y con los brazos para el cerco. La madera } \\
\text { se usa para artesanías. (Pedro B., } 52 \text { años, Varón). }\end{array}$ & $\begin{array}{l}\text { Son los relacionados con la ornamentación y comercialización. } \\
\text { Se destacan por una cuestión estética, lo cual aumenta su } \\
\text { valor comercial. } \\
\text { Los tengo de adorno, también los vendo, dicen que hay que } \\
\text { sacar un permiso, por ser cosas como de patrimonio que no se } \\
\text { pueden vender pero no se. (Rosa P., } 54 \text { años, Mujer)- } \\
\text { Estas son para vender, a la gente les gusta los bochitas } \\
\text { [género Gymnocalycium]. (Rosa M., } 50 \text { años, Mujer) } \\
\text { Los cactus, son para adorno, yo los traigo para mostrar, por } \\
\text { donde voy traigo. (Rosa Q., } 80 \text { años, Mujer) } \\
\text { La valoración se resalta como negativa por el daño que } \\
\text { producen a los animales. } \\
\text { Son malos porque lastiman a los animales, porque el puqui } \\
\text { [espinas] queda encarnado en el animal. (Nini R. } 72 \text { años, } \\
\text { Varón) } \\
\text { Sin embargo se valora por una cuestión simbólica y de } \\
\text { identidad cultural. Además, aquellos pobladores de mayor } \\
\text { edad y los que tienen familiares de varias generaciones } \\
\text { anteriores que estuvieron en ese campo, mencionan una } \\
\text { pérdida de conocimientos ligada al avance del desmonte y de } \\
\text { la urbanización que sufrieron las sierras. } \\
\text { Ahora se está perdiendo todo, yo soy nacido y criado en } \\
\text { el campo, todo lo que hay en el monte sirve para algo, los } \\
\text { cactus todos se usan, todos los yuyos [plantas herbáceas] } \\
\text { son remedios. Se está perdiendo todo por como desmontan, } \\
\text { antes habia cualquier cantidad pero ahora no porque se limpió } \\
\text { todo, y es bueno que haya un buen monte, ahora llueve y se } \\
\text { inunda todo porque el agua no tiene donde quedarse. (Jesús } \\
\text { G., } 68 \text { años, Varón) }\end{array}$ \\
\hline
\end{tabular}




\section{J. K. Torrico Chalabe y C. Trillo - Conocimientos, valoración y usos de Cactáceas}

usos y las prácticas tradicionales son menores, siendo las mismas personas pertenecientes a esta unidad quienes plantean que se han "olvidado" o que los recuerdan como anécdotas. Esta dinámica en los saberes coincide con el avance de la urbanización y la consolidación del área como zona turística, que algunos informantes destacan como parte de los factores de pérdida de conocimientos y usos ligados a tradiciones. Estos cambios permiten el desarrollo de nuevas actividades económicas, asociadas en las narrativas a la aparición de los nuevos usos (principalmente el ornamental); en este sentido, podría compararse con los resultados de Muiño (2012) que reflejan similares patrones de uso de los recursos en la provincia de La Pampa. Es importante destacar que si bien no contamos con datos ecológicos que permitan concluir sobre una pérdida de la biodiversidad en las zonas del Sistema Serrano, los mismos entrevistados relacionan estas transformaciones al desmonte, como destacan Arias Toledo et al. (2010) para los bosques chaqueños de Córdoba. Por otro lado, en esta zona se reconocen y valoran más los usos asociados a la comercialización y al rol ornamental, de las mismas especies que en Salinas Grandes presentan usos tradicionales. De esta manera es posible afirmar la existencia de un proceso de resignificación de los usos, generado principalmente por ciertas especies vegetales que pasan del contexto tradicional al no tradicional, en el que muchos usos originales no se transponen de modo exacto; por el contrario, sufren modificaciones (Hurrell et al., 2013). La resignificación refleja la compleja construcción de respuestas de ajuste a diferentes condiciones bioculturales (ecológicas y económicas), a las que se ven expuestos los criollos, pequeños productores y campesinos con los que se trabajó en el Noroeste de Córdoba. Por un lado, los pobladores de Salinas Grandes, que viven en condiciones de aridez extrema y con escasos recursos herbáceos, conservan conocimientos ligados a tradiciones que son necesarios para asegurar su reproducción social, donde las Cactáceas aparecen como recursos claves para su subsistencia. Por otra parte, los del Sistema Serrano, que cuentan con mayor diversidad de recursos forrajeros herbáceo, arbustivo y arbóreo, $\mathrm{y}$ menor distancia a las zonas urbanas, pueden ofrecer servicios comerciales y reorientan sus conocimientos hacia nuevas actividades, si bien las Cactáceas siguen teniendo un rol protagónico.
En este caso, se evidencia la flexibilidad de estos saberes (dentro del CEL) que permite ajustes a las situaciones de cambio contextual.

En concordancia con otras publicaciones (Fernández-Llamazares \& Reyes-García, 2016), este trabajo permite contribuir a la discusión y a una nueva comprensión sobre las dinámicas que tiene el CEL y su rol en un contexto de cambio.

\section{Contribución de las AUtORAS}

Las autoras han realizado conjuntamente y a partes iguales la colecta de datos, su interpretación y redacción del manuscrito.

\section{Agradecimientos}

Agradecemos a las personas que colaboraron con este trabajo, tanto las de Salinas Grandes y del Sistema Serrano que compartieron sus historias de vida y percepciones, y a J. A. Hurrell, P. Demaio, L. Ahumada, V. Saur Palmieri, C. Ibarra y G. Tortoni, quienes nos ayudaron en la realización de esta contribución. A los dos revisores anónimos por sus sugerencias. También hacemos llegar nuestro reconocimiento al CONICET. Este trabajo se financió en el marco del Proyecto de Investigación de la Secretaria de Ciencia y Técnica - Universidad Nacional de Córdoba (2016-2017) "Percepción, manejo y uso de unidades de paisajes en zonas rurales de Córdoba, transformaciones en el tiempo", Dirigido por la Dra. Trillo C.

\section{Bibliografía}

AGUILERA, R. M., A. DURAND-SMITH, E. M. RODRÍGUEZ, \& M. ROMERO MENDOZA. 2003. Veinticinco años de investigación cualitativa en salud mental y adicciones con poblaciones ocultas. Primera parte. Salud Mental 26: 76-83.

AHUMADA, M. L., \& C. TRILLO. 2017. Diversidad de Especies Naturalizadas del género Opuntia (Cactaceae) utilizadas por los pobladores de Córdoba (Argentina). Bol. Soc. Argent. Bot. 52: 193-208.

ALBUQUERQUE, U. P. DE, L. V. F. CRUZ DA CUNHA, R. F. P. LUCENA, \& R. R. N. ALVES. 2014. Methods and Techniques in Ethnobiology and Ethnoecology. Springer-Humana Press, New York, USA. 
ALBUQUERQUE, U. P., PAIVA DE LUCENA, R. F., FERNANDES CRUZ DA CUNHA, L. V. \& R. R. NÓBREGA ALVES. 2018. Methods and Techniques in Ethnobiology and Ethnoecology. 2nd ed. Springer-Humana Press, New York, USA

ALDRIGE, A., \& K. LAVINE. 2003. Topografia del mundo social. Teoría y práctica de la investigación mediante encuestas. Gedisa Editorial, Barcelona, España.

ARENAS, P., \& G. F. SCARPA. 1998. Ethnobotany of Stetsonia coryne (Cactaceae), the "cardón" of the Gran Chaco. Haseltonia 6: 41-51.

ARIAS TOLEDO B. \& C. TRILLO. 2014. Animales y plantas que curan: avances sobre la farmacopea natural de los pobladores del área de Laguna Mar Chiquita. Revista de la Facultad de Ciencias Exactas, Físicas y Naturales 1(2):77-85.

ARIAS TOLEDO, B., C. TRILLO \& M. GRILLI. 2010. Uso de plantas medicinales en relación al estado de conservación del bosque en Córdoba, Argentina. Ecología austral 20:235-246.

ATLAS DE LOS BOSQUES NATIVOS ARGENTINOS. 2003. Proyecto Bosques Nativos y Áreas Protegidas BIRF 4085-AR. Dirección de Bosques, Secretaría de Ambiente y Desarrollo Sustentable, Buenos Aires, Argentina.

BERBERIÁN, E. 1987. Crónicas del Tucumán: siglo XVI. Editorial Comechingonia, Córdoba, Argentina.

BERGAMÍN, G. 1992. Conceptualización sobre marginalidad, tipología de productores y desarrollo para caracterizar el Chaco árido. En: KARLIN, U. \& R. COIRINI (Eds). Sistemas agroforestales para pequeños productores de zonas áridas; pp. 13-17. Proyecto de desarrollo agroforestal en comunidades rurales del noroeste argentino, Universidad Nacional de Córdoba, Argentina.

BERKES, F. 1999. Context of Traditional Ecological Knowledge. In: Berkes F. (Ed.) Sacred Ecology, Traditional Ecological Knowledge and Resource Management, pp. 3-15. Taylor \& Francis, Philadelphia, USA.

BERNARD, H. R. 1995. Research Methods in Anthropology Qualitative and Quantitative Approaches. Altamira Press, Oxford, UK.

BIURRUN, E., L. GALETTO, A. M. ANTON \& F. BIURRUN. 2007. Plantas silvestres comestibles utilizadas en poblaciones rurales de la Provincia de La Rioja (Argentina). Kurtziana 33: 121-140.

CABAllERO, J. \& L. CORTÉS. 2001. Percepción, uso y manejo tradicional de los recursos vegetales en México. En: RENDÓN, B., S. REBOLLAR, J. CABALLERO \& M. A. MARTÍNEZ (Eds.). Plantas, Cultura y Sociedad: Estudio sobre la relación entre seres humanos y plantas en los albores del siglo XXI, pp. 79-100. Editorial Universidad Autónoma de México, Distrito Federal, México.
CABAllero, J., A. CASAS, L. CORTÉS \& C. MAPES. 1998. Patrones en el conocimiento, uso y manejo de plantas en pueblos indígenas de México. Estud. Atacameños 16: 181-196.

CABIDO, M. \& M. J. PACHA. 2002. Vegetación y Flora de la Reserva Natural Chancaní. Publicaciones Técnicas serie C. Agencia Córdoba Ambiente, Córdoba, Argentina.

CABIDO, M. \& M. ZAK. 1999. Vegetación del Norte de Córdoba. Secretaría de Agricultura, Ganadería y Recursos Renovables de la provincia de Córdoba y Agencia Córdoba Ambiente, Córdoba, Argentina.

CABIDO, M., M. ZAK, A. CINGOLANI, D. CÁCERES \& S. DÍAZ. 2005. Cambios en la cobertura de la vegetación del centro de Argentina: ¿Factores directos o causas subyacentes? En: OESTERHELD, M., M. AGUIAR, C. GHERSA \& J. PARUELO (Eds.). La heterogeneidad de la vegetación de los agroecosistemas, pp. 271-300. Universidad Nacional de Buenos Aires, Buenos Aires, Argentina.

CABRERA, A. 1976. Regiones fitogeográficas argentinas. En: KUGLER W. (Ed.). Enciclopedia Argentina de Agricultura y Jardinería, pp. 1-85. Segunda Edición. Editorial ACME, Buenos Aires, Argentina.

CÁCERES, D. 2014. Amenazas y desafíos que enfrenta el campesinado en Argentina. ¿Descampesinización o Persistencia? En: CRAVIOTTI C. (Ed.). Agricultura Familiar en Latinoamérica. Continuidades, Transformaciones y Controversias, pp. 205-232. Editorial Ciccus, Buenos Aires, Argentina.

CÁCERES, D., F. SILVETTI, G. FERRER \& G. SOTO. 2006. Y... vivimos de las cabras. Transformaciones sociales y tecnológicas de la capricultura. La Colmena, Buenos Aires, Argentina.

CÁCERES, D., G. SOTO, G. FERRER, F. SILVETTI \& C. BISIO. 2010. La expansión de la agricultura industrial en Argentina Central. Su impacto en las estrategias campesinas. Cuadernos de Desarrollo Rural 7: 91-119.

CASAS, A. 2001. Silvicultura y Domesticación de plantas en Mesoamérica. En: RENDÓN, B., S. REBOllaR, J. CABAllero \& M. A. MARTÍNEZ (Eds.). Plantas, Cultura y Sociedad: Estudio sobre la relación entre seres humanos y plantas en los albores del siglo XXI, pp. 123157. Editorial Universidad Autónoma de México, Distrito Federal, México.

CASAS, A., J.L. VIVEROS, E. KATZ \& J. CABALLERO. 1987. Las plantas en la alimentación mixteca: una aproximación etnobotánica. América indígena 47:317-343.

Catálogo de Plantas Vasculares del Conosur: http:// www2.ddarwin.edu.ar/Proyectos/FloraArgentina/ FA.asp. 


\section{J. K. Torrico Chalabe y C. Trillo - Conocimientos, valoración y usos de Cactáceas}

CAVANNA, J. A., G. CASTRO, R. COIRINI, U. KARLIN \& M. KARLIN. 2009. Caracterización socio-productiva de ocho comunidades de pequeños productores de las Salinas Grandes, provincia de Catamarca, Argentina. Multequina 18: 15-29.

CAVANNA, J., G. CASTRO, U. KARLIN \& M. KARLIN. 2010. Ciclo ganadero y especies forrajeras en Salinas Grandes, Catamarca, Argentina. Zonas Áridas 14: 170-180.

COIRINI, R. O., M.S. KARLIN \& G.J. REATI. 2010. Manejo sustentable del ecosistema Salinas Grandes, Chaco árido. Grupo Editor Encuentro, Córdoba, Argentina.

CORREA, S. L., S. TURBAY \& M. VÉLEZ. 2012. Conocimiento ecológico local sobre ecosistemas marinos en dos comunidades costeras: El Valle y Sapzurro. Gestión y Ambiente 15: 17-32.

COSTA-NETO, E. M. 2002. Manual de Etnoentomología. Editorial SEA Zaragoza, Bahia, Brasil.

COTTON, C. M. 1996. Ethnobotany: principles and applications. John Wiley \& Sons, New York, USA.

DEMAIO, P. \& M. TREVISSON. 2006. Cactus de Córdoba y el centro de Argentina. Editorial L.O.L.A. Literature of Latin America. Buenos Aires, Argentina.

DI LULLO, O. 1946. Contribución al estudio de las voces santiagueñas. Santiago del Estero. Publicaciones de la Provincia de Santiago del Estero.

FAO. 2007. Las tierras áridas del mundo. En: Organización de las Naciones Unidas para la Alimentación y la Agricultura (Ed.), Secuestro de carbono en tierras áridas: Informes sobre recursos mundiales del suelo, pp. 9-19, Roma, Italia.

FERNÁNDEZ-LLAMAZARES, A. \& V. REYESGARCÍA. 2016. An Ethnobiology of Change. In: ALBUQUERQUE U.P. \& R.R. NÓBREGA ALVES (Eds.). Introduction of Ethnobiology, pp 69-74. Springer International Publishing.

FERNANDES SARAIVA, R. C. 2012. Saberes, fazeres e natureza nas vozes de mulheres da Chapada dos Veadeiros-Goias. História Oral 1: 209-229.

HARO, J. G., P. MICHELUTTI, R. M. TORRES, A. MOLLI \& E. H. BUCHER. 2006. Mamíferos. En: BUCHER, E. H. (Ed.). Bañados del río Dulce y Laguna Mar Chiquita, pp. 277-283. Academia Nacional de Ciencias, Córdoba, Argentina.

HERRERA， D.R. 2003. Tuna: Planificación e Implantación del Tunal Comercial. Instituto Nacional Tecnológico Argentino, Centro Regional Córdoba, Oficina Técnica Villa de María, Córdoba, Argentina.

HOYOS, L. E., A. M. CINGOLANI, M. ZAK, M.V. VAIERETTI, D.E. GORLA \& M.R. CABIDO. 2012. Deforestation and precipitation patterns in the arid Chaco forests of central Argentina. Appl. Veg. Sci. 16: 260-271.
HURRELL, J. A., STAMPELLA, P. C., DOUMECQ, M. B. \& POCHETTINO, M. L. 2018. Ethnoecology in Pluricultural Contexts: Theoretical and Methodological Contributions. In: ALBUQUERQUE, U. P., PAIVA DE LUCENA, R. F., FERNANDES CRUZ DA CUNHA, L. V. \& R. R. NÓBREGA ALVES (eds.), Methods and Techniques in Ethnobiology and Ethnoecology, pp. 163-186. 2nd ed. Springer-Humana Press, New York, USA

HURRELL, J. A., M.L. POCHETTINO, J.P. PUENTES \& P.M. ARENAS. 2013. Del marco tradicional al escenario urbano: Plantas ancestrales devenidas suplementos dietéticos en la conurbación Buenos Aires-La Plata, Argentina. BLACPMA 12: 499-515.

HURRELL, J. A. 2014. Urban Ethnobotany in Argentina: Theoretical advances and methodological strategies. Ethnobio. Conserv. 3(2):1-11.

INDEC. 2010. Censo Nacional de Población, Hogares y Viviendas. Argentina. Disponible en: www.indec. gov.ar [Acceso: abril 2018].

KARLIN, M. S., U.O. KARLIN, R.O. COIRINI, G.J. REATI \& R.M. ZAPATA. 2013. El Chaco Árido. Universidad Nacional de Córdoba, Córdoba, Argentina.

KIESLING, R. \& O .E. FERRARI. 2005. 100 Cactus Argentinos. Editorial Albatros Saci Buenos Aires, Argentina.

KIMBRELL, A. (Ed.). 2002. The fatal harvest reader: the tragedy of industrial agriculture. Island Press, Washington, USA.

MAFFI, L. 2001. Introduction. On the interdependence of biological and cultural diversity. In: MAFFI, L. (Ed.) Biocultural diversity: Linking language, knowledge, and the environment, pp. 1-50. Smithsonian Inst., Washington.

MAFFI, L. 2005. Linguistic, cultural, and biological diversity. Annu Rev Anthropol 29: 599-617.

MARTÍNEZ-CROVETTO, R. 1964. Estudios etnobotánicos. I: Nombres de plantas y su utilidad, según los indios tobas del este del chaco. Bonplandia 1: 279-333.

MUIÑO, W. 2012. Los "puestos" del oeste pampeano. La relación de los campesinos criollos con las plantas a través de los huertos y jardines. En: ARENAS P. (Ed.) Etnobotánica en zonas áridas y semiáridas del Cono Sur de Sudamérica, pp. 225-250. CEFYBOCONICET, Buenos Aires, Argentina.

NAZAREA, V. D. 1999. A view from a point: ethnoecology as situated knowledge. In: NAZAREA, V. D. (ed), Ethnoecology: situated knowledge/located lives, pp 3-20. University of Arizona Press, Tuscon, AZ.

PADUA, J. 1994. Técnicas de investigación aplicadas a las ciencias sociales. Fondo de Cultura Económica, México. 
PARLEE, B. L., K. GEERTSEMA \& A. WILLIER. 2012. Social-ecological thresholds in a changing boreal landscape: insights from Cree knowledge of the Lesser Slave Lake region of Alberta, Canada. Ecol. Soc. 17: 20.

PASTOR, S., M. MEDINA, A. RECALDE, L. LÓPEZ \& E. BERBERIÁN. 2012. Arqueología de la región montañosa central de Argentina. Avances en el conocimiento de la historia Prehispánica tardía. Relaciones 37: 89-112.

POCHETTINO, M.L. \& J.A. HURRELL. 2013. Los cambios ambientales en la región rioplatense (Argentina) y las narrativas de los pobladores locales. Actas X Reunión de Antropología del Mercosur, Córdoba, Argentina.

RECALDE, A. 2015. Representaciones en contexto. Características del Paisaje Rupestre de Cerro Colorado (Sierras Del Norte, Córdoba, Argentina). Relaciones 30: 523-548.

ROIG, F. A. \& J.R. BÁRCENA. 1983. Tephrocactus andicola (Cactaceae), recurso alimenticio de aborígenes pre y posthispánicos de Mendoza, Argentina. Parodiana 2: 59-66.

RUIZ POSSE, E., U.O. KARLIN, E. BUFFA, M. KARLIN, C.G. LEVRA \& G. CASTRO. 2007. Ambientes de las salinas grandes de Catamarca, Argentina. Multequina 16: 123-137.

SCARPA, G. F. 2007. Etnobotánica de los Criollos del oeste de Formosa: Conocimiento tradicional, valoración y manejo de plantas forrajeras. Kurtziana 33: 153-174.
SOLAE. 2016. Código de Ética. Revista Etnobiología 14.

TORRICO CHALABE, J. K. \& C. TRILLO. 2015. Prácticas de manejo, usos y valoración de taxones de Cactaceae en el noroeste de Córdoba, Argentina. Bonplandia 24: 5-22.

TRILlO, C., P. DEMAIO, S. COLANTONIO \& L. GALETTO. 2007. Conocimiento actual de plantas tintóreas por los pobladores del valle de Guasapampa, provincia de Córdoba. Kurtziana 33: 65-71.

TRILLO, C. 2010. Valoración del bosque y conocimiento de las plantas silvestres por parte de los pobladores de las Sierras de Guasapampa, Noroeste de la Provincia de Córdoba. Tesis Doctoral, Facultad de Ciencias Exactas, Físicas y Naturales, Universidad Nacional de Córdoba, Córdoba. Argentina. Pp. 188.

VANDEBROEK, I., V. REYES-GARCÍA, U.P. DE ALBUQUERQUE, R. BUSSMANN \& A. PIERONI. 2011. Local knowledge: Who cares? Journal of Ethnobiology and Ethnomedicine 7: 35.

ZAPATA, R.M., U.O. KARLIN, F. LUCERO, R.O. COIRINI \& M. KARLIN. 2005. Manejo Sustentable del Ecosistema Salinas Grandes. Manejo de los tunales. Proyecto "Desarrollo de un polo productivo integral en el norte de Córdoba: cultivo y procesamiento de la tuna (Opuntia ficusindica) y especies aromáticas bajo certificación orgánica". Proyectos Federales de Innovación Productiva del Ministerio de Ciencia, Tecnología e Innovación Productiva, Córdoba, Argentina. 